\title{
INFLUENCIA DEL ORIGEN DE LAS SEMILLAS EN EL DESEMPEÑO DE COIGÜE (Nothofagus dombeyi (Mirb.) Oerst.) EN ENSAYOS DE 15 AÑOS EN LA COSTA Y PRECORDILLERA DE LA REGION DE LOS RIOS
}

\author{
Gutiérrez, Braulio ${ }^{3}$
}

\section{RESUMEN}

Se caracteriza el desempeño de coigüe, en términos de supervivencia, altura, diámetro y rectitud de fuste a los 14-15 años de edad en dos ensayos establecidos en costa y pre cordillera de la región de Los Ríos Chile (Huillilemu en San José de la Mariquina y Remeco en Panguipulli); se analiza también el efecto del origen de las semillas (zonas de procedencias) sobre las variables mencionadas.

Se constata un crecimiento similar en ambos ensayos y de la misma magnitud que los indicados para otras plantaciones de coigüe. La supervivencia y rectitud de fuste es mayor en Remeco que en Huillilemu, aun cuando en ambos casos predominan árboles de escasa rectitud.

No se detectan diferencias de desempeño asociadas al efecto de las zonas de procedencias, concluyéndose y respaldándose con antecedentes bibliográficos una escasa diferenciación genética entre poblaciones de coigüe. Esta escasa diferenciación obedecería al efecto homogeneizador del flujo génico presente en las extensas y relativamente continuas poblaciones de coigüe.

Palabras clave: Nothofagus dombeyi, coigüe, procedencias, crecimiento, forma.

\section{SUMMARY}

The performance of coigüe, in terms of survival, height, diameter and straightness of the stem at 14-15 years of age in two trials established in the coast and pre-mountain range of the Los Ríos Chile region (Huillilemu in San José de la Mariquina and Remeco in Panguipulli) is characterized; The effect of the origin of the seeds (areas of provenance) on the mentioned variables is also analyzed.

Both trials show a similar growth and it is of the same magnitude as those indicated for other coigüe plantations. The survival and straightness of the stem is greater in Remeco than in Huillilemu, even though in both cases trees with little straightness predominate.

No differences in performance associated with the effect of the areas of provenance were detected, concluding and supporting with bibliographic antecedents a scarce genetic differentiation between populations of coigüe. This scarce differentiation would be due to the homogenizing effect of the gene flow present in the extensive and relatively continuous populations of coigüe.

Keywords: Nothofagus dombeyi, coigüe, provenance, growth, stem shape.

\footnotetext{
${ }^{3}$ Ingeniero Forestal. Instituto Forestal, Sede Bio Bio. bgutierr@infor.cl
} 


\section{INTRODUCCIÓN}

Si bien se registran crecimientos bastantes heterogéneos, se reconoce que coigüe (Nothofagus dombeyi) manifiesta un crecimiento más bien rápido entre las especies arbóreas del cono sur (Cruz-Johnson, 2013). En general, su crecimiento en altura es mayor que el observado en otras especies del género Nothofagus, reflejando así la alta productividad tradicionalmente asociada a los rodales dominados por coigüe (Esse et al., 2014). En plantaciones también presenta un alto crecimiento, incluso superior al exhibido en bosques naturales.

Como ejemplo de lo anterior, Donoso y Soto (2010) indican que en plantaciones con silvicultura intensiva de establecimiento puede alcanzar incrementos medios anuales de hasta 1,9 $\mathrm{cm}$ en diámetro y $1,5 \mathrm{~m}$ en altura. Esta atractiva tasa de crecimiento ha determinado que entre las especies nativas que se utilizan en plantaciones forestales, coigüe sea una de las más difundidas.

Considerando la extensa distribución natural de coigüe, puede existir una gran distancia y diferencia de condiciones ambientales y ecológicas entre el sitio donde se producen las semillas y el lugar donde realiza la plantación. Por lo mismo, resulta especialmente interesante generar información para orientar y facilitar las decisiones de silvicultores y forestadores respecto a orígenes de semilla que contribuyan por una parte a aumentar rendimiento de la plantación, y por otra eviten que esa plantación se constituya en una fuente de contaminación genética para las poblaciones naturales existentes en esa área.

Una consideración prudente es el uso de semilla local, aunque como se indica en Gutiérrez (2019) debe tenerse en cuenta que no siempre el origen local es el más adecuado, y que hay situaciones donde puede ser necesario o aconsejable utilizar orígenes foráneos.

El enfoque tradicional para contribuir al ordenamiento genético territorial respecto al uso y movimiento de semillas lo constituyen las regiones o zonas de procedencia. Enfoques alternativos, como el de zonas genéticas, son complementarios y se diferencian del mismo por considerar información de marcadores moleculares para definir las diferentes zonas (Pastorino y Mondino, 2016; Azpilicueta et al., 2016; Hasbún et al., 2014).

El modelamiento de nicho ecológico es también una alternativa para predecir los sitios donde los distintos orígenes de semillas podrían prosperar adecuadamente (Alía et al., 2009b; Hasbún et al., 2014; Marchelli et al., 2016). No obstante, por su simplicidad, las zonas de procedencia han sido el mecanismo más utilizado, estas corresponden a un conjunto de territorios sometidos a condiciones ecológicas suficientemente uniformes, en los que se desarrollan poblaciones que presentan características fenotípicas o genéticas análogas (Agúndez et al., 1995; Alía et al., 2009a; Garcia del Barrio et al., 2001).

El sustento de estas zonas se basa en que las diferencias entre poblaciones de una misma especie que ocupan distintos territorios, si bien se pueden atribuir en parte a la expresión de plasticidad fenotípica, obedecen también a diferencias que puede tener una base genética producto de procesos de selección acaecidos durante múltiples generaciones.

En Chile Hasbún et al. (2014) efectuaron una propuesta inicial de zonas de procedencia para coigüe basada en las 7 grandes áreas (estratos) definidas por Alarcón et al. (2013) en base a herramientas de modelamiento ecológico.

Esta zonificación fue posteriormente descartada en función de estudios moleculares que no indicaban mayor diferenciación genética entre ellas. Por su parte, Quiroz y Gutiérrez (2014), en una propuesta de reglamento para regular la producción, comercio y certificación de semillas y 
plantas forestales, elaboraron un mapa de zonas de procedencia para especies forestales nativas, pero que no ha sido validado con estudios moleculares.

En el presente artículo se utiliza la clasificación de zonas de procedencias de Quiroz y Gutiérrez (2014) para evaluar el efecto del origen de las semillas, en términos de zona de procedencia, sobre variables de supervivencia, crecimiento y rectitud de fuste de individuos de coigüe de 14 y 15 años de edad establecidos en dos ensayos de procedencias y progenies establecidos en las cercanías de las localidades de San José de la Mariquina y de Neltume, en la región de Los Ríos, Chile:

\section{OBJETIVO}

Determinar la existencia de diferencias atribuibles a las zonas de procedencias y orientar respecto a los orígenes y materiales idóneos para utilizar en las áreas afines a cada zona de ensayo.

\section{MATERIAL Y MÉTODO}

Los ensayos considerados corresponden a las pruebas de procedencias y progenies de coigüe Huillilemu y Remeco.

El ensayo Huillilemu se ubica en el predio del mismo nombre, en la comuna de San José de la Mariquina, región de Los Ríos, $\left(39^{\circ} 33,167^{\prime} \mathrm{S} ; 7^{\circ} 4,356^{\prime}\right.$ O) a una altitud de $23 \mathrm{msnm}$, en un área de clima templado lluvioso, clasificada dentro del subtipo clima de costa occidental con influencia mediterránea y caracterizado por las altas precipitaciones, con un promedio anual de $2.400 \mathrm{~mm}$ (García et al., 2013).

El ensayo considera 5 bloques completos al azar donde se prueban 73 progenies correspondientes a 23 procedencias agrupadas en 11 zonas de procedencias. Los datos evaluados corresponden a la medición efectuada a los 15 años de edad.

El ensayo Remeco se ubica en el predio homónimo, en las cercanías de la localidad de Neltume (3949,699'S; $\left.71^{\circ} 57,366^{\prime} \mathrm{O}\right)$, en la comuna de Panguipulli, provincia de Valdivia, región de Los Ríos, a $512 \mathrm{msnm}$, en un área con clima de tendencia oceánica, de carácter templado-húmedo con registro de precipitaciones durante todo el año y ausencia de déficit hídrico. Las precipitaciones anuales alcanzan a unos $2.000 \mathrm{~mm}$, las que se registran tanto en forma de lluvia como de nieve (Teillier et al., 2013).

El ensayo considera 5 bloques completos al azar donde se prueban 67 progenies correspondientes a 17 procedencias agrupadas en 9 zonas de procedencias. Los datos evaluados corresponden a la medición efectuada a los 14 años de edad.

Las variables evaluadas fueron supervivencia $(S)$, altura total $(H)$, diámetro a la altura del pecho (DAP) y rectitud de fuste (RF). Esta última variable categorizada en una escala cualitativa compuesta por los cuatro niveles que se ilustran en la Figura $\mathrm{N}^{\circ} 1$. 


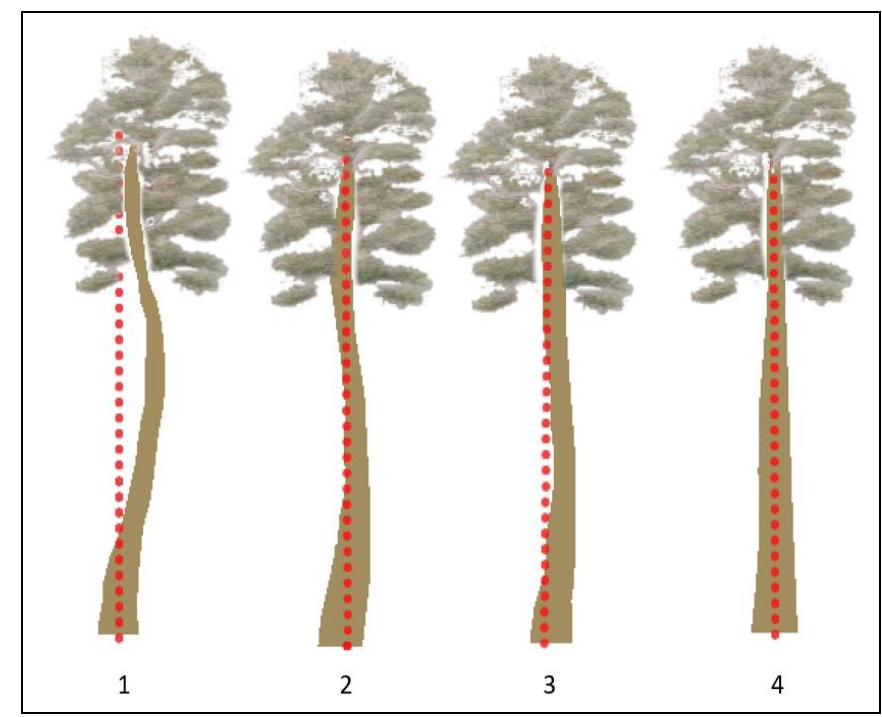

Figura $\mathrm{N}^{\circ} 1$

ESCALA CUALITATIVA PARA EVALUACIÓN DE RECTITUD DE FUSTE

Se calcularon los parámetros medios y de dispersión del ensayo para cada variable evaluada y se efectuó análisis de varianza para verificar la existencia de diferencias estadísticamente significativas para cada una de las variables a nivel de zonas de procedencia.

Los análisis de varianza se complementaron con la prueba de Tuckey para comparación múltiple de medias (alfa $=0,05$ ), usando la diferencia mínima significativa (DMS) como criterio para decidir la existencia de diferencias estadísticamente significativas para cada variable en función de las zonas de procedencia analizadas.

En el caso de la variable supervivencia los porcentajes fueron transformados a unidades de Bliss, posteriormente para efectos de presentar los resultados, las DMS fueron revertidas nuevamente a porcentajes.

Para el análisis a nivel de zonas de procedencia, se utilizó el mapa desarrollado por Quiroz y Gutiérrez (2014) en el marco de una propuesta de reglamento para normar y regular el origen de las semillas de especies forestales nativas. Este mapa corresponde a una segmentación divisiva y multiespecífica, donde se divide el área de interés silvícola del país, desde la región de Coquimbo a la de Magallanes, en 22 zonas de procedencias relativamente homogéneas en base a información de clima, fisiografía, vegetación y suelos.

En la Figura $\mathrm{N}^{\circ} 2$ y Cuadro $\mathrm{N}^{\circ} 1$ se muestran y describen las zonas de procedencia desde donde se obtuvo la semilla de coigüe que dio origen los ensayos evaluados. En esta clasificación el ensayo Huillilemu se ubica en la zona de procedencia ZP-15, en tanto el ensayo Remeco está establecido en la zona de procedencias ZP-14. 


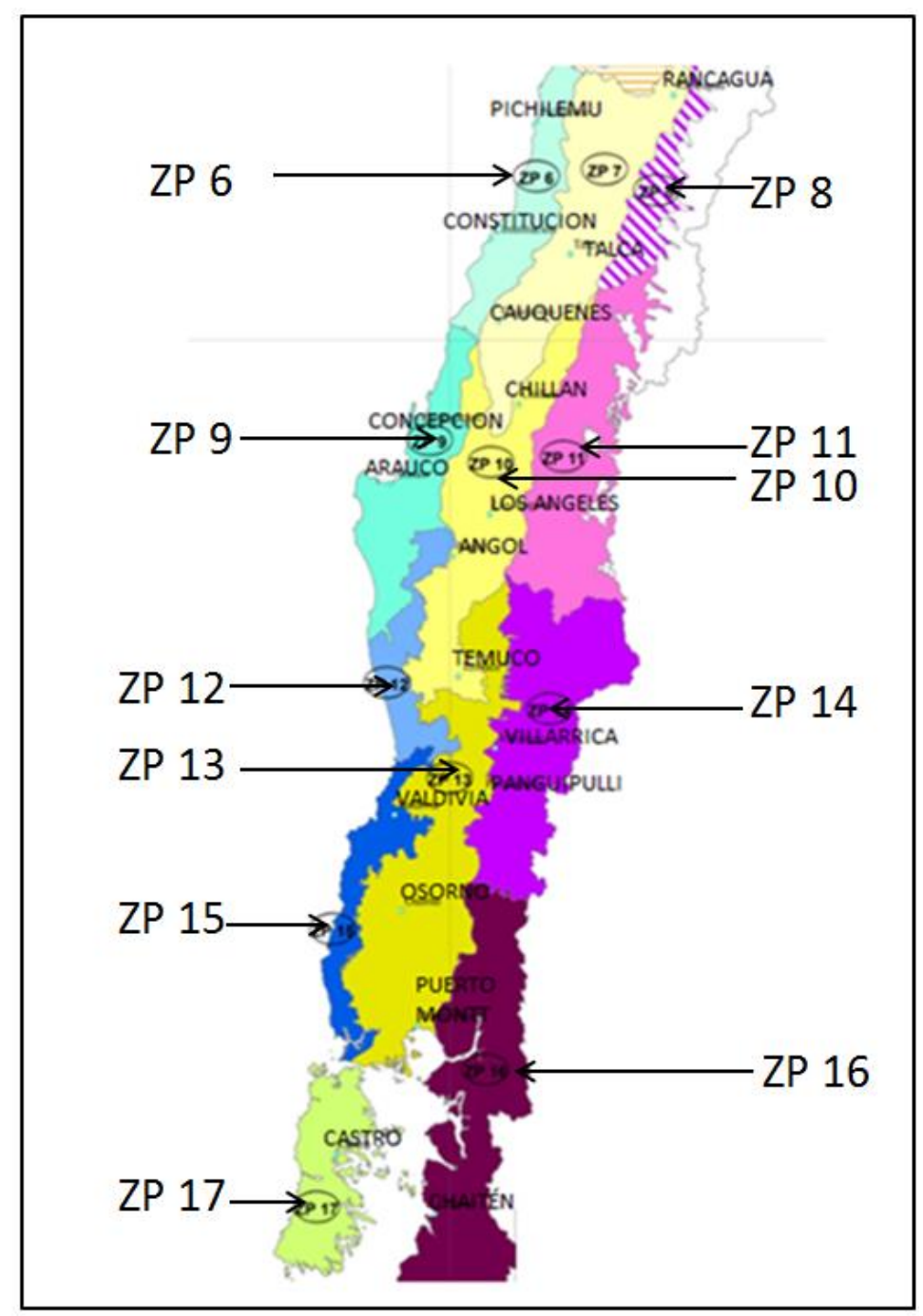

Figura $\mathrm{N}^{\circ} 2$

DEFINICIÓN DE ZONAS DE PROCEDENCIAS UTILIZADA PARA INDIVIDUALIZAR LAS PROCEDENCIAS EVALUADAS EN EL ENSAYO 


\section{Cuadro $\mathrm{N}^{\circ} 1$ \\ DESCRIPCIÓN DE LAS ZONAS DE PROCEDENCIAS CONSIDERADAS EN LOS ENSAYOS DE COIGÜE DE HUILLILEMU Y REMECO}

Zonas de Procedencia, Nombre y Descripción

ZP-6. COSTA HUASA: Límites $33^{\circ} 55^{\prime} \mathrm{S} 71^{\circ} 50^{\prime} \mathrm{W}, 36^{\circ} 05^{\prime} \mathrm{S} 72^{\circ} 30^{\prime} \mathrm{W}$; costa regiones O'Higgins y Maule; altitudes entre 0 y $600 \mathrm{msnm}$; rango de precipitación media anual 501-1000 mm; presencia de tipos forestales Roble-Hualo, Esclerófilo y Roble-Rauli-Coihue; material parental del suelo: roca metamórfica terreno intermedio alto.

ZP-8. PLANCHON: Límites $33^{\circ} 55^{\prime} \mathrm{S} 71^{\circ} 34^{\prime} \mathrm{W}, 35^{\circ} 30^{\prime} \mathrm{S} 70^{\circ} 51^{\prime} \mathrm{W}$; pre cordillera y cordillera de Los Andes regiones de O'Higgins y Maule; altitudes entre 800 y 1500 msnm; rango de precipitación media anual 501 $1500 \mathrm{~mm}$; presencia de tipos forestales Roble-Raulí-Coihue, Esclerófilo, Ciprés de la Cordillera y Lenga; descripción del suelo: sedimentos antiguos de origen volcánico, afloramientos rocosos.

ZP-9. GOLFO DE ARAUCO: Límites $36^{\circ} 01^{\prime} \mathrm{S} 72^{\circ} 46^{\prime} \mathrm{W}, 38^{\circ} 27^{\prime} \mathrm{S} 73^{\circ} 17^{\prime} \mathrm{W}$; costa región del Biobío; altitudes entre 0 y 600 msnm; rango de precipitación media anual $1001-1500 \mathrm{~mm}$; presencia de tipos forestales Roble-Rauli-Coihue, Esclerófilo, Roble-Hualo y Araucaria; descripción del suelo: sedimentos marinos en posiciones bajas e intermedias, rocas graníticas en terrenos altos.

ZP-10. SALTO DEL LAJA: Límites $36^{\circ} 09^{\prime} \mathrm{S} 72^{\circ} 31^{\prime} \mathrm{W}, 38^{\circ} 55^{\prime} \mathrm{S} 72^{\circ} 19^{\prime} \mathrm{W}$; depresión intermedia regiones del Maule, Biobío y Araucanía; altitudes entre 200 y 700 msnm; rango de precipitación media anual 1001-1500 $\mathrm{mm}$; presencia de tipos forestales Esclerófilo, Roble-Raulí-Coihue; descripción del suelo presente en esta zona: rocas graníticas en terrenos topografía intermedia, arenas volcánicas en terrenos planos a ligeramente ondulados, cenizas volcánicas, y suelos rojos arcillosos.

ZP-11. NEVADOS DE CHILLÁN: Límites $35^{\circ} 21^{\prime} \mathrm{S} 70^{\circ} 28^{\prime} \mathrm{W}, 37^{\circ} 56^{\prime} \mathrm{S} 72^{\circ} 07^{\prime} \mathrm{W}$; pre cordillera y cordillera de Los Andes regiones del Maule y Biobío; altitudes entre 700 y 1400 msnm; rango de precipitación media anual 1501-2500 mm; presencia de tipos forestales Roble-Raulí-Coihue, Lenga, Roble-Hualo, Ciprés de la Cordillera y Araucaria; descripción del suelo cenizas volcánicas modernas en terrenos intermedios a altos.

ZP-12. NAHUELBUTA: Límites $37^{\circ} 38^{\prime} \mathrm{S} 73^{\circ} 04^{\prime} \mathrm{W}, 39^{\circ} 18^{\prime} \mathrm{S} 72^{\circ} 37^{\prime} \mathrm{W}$; costa e interior región de la Araucanía; altitudes entre 0 y $600 \mathrm{msnm}$; rango de precipitación media anual 1501-2000 mm; presencia de tipos forestales Roble-Raulí-Coihue, Siempreverde y Araucaria; descripción del suelo: sedimentos marinos en posiciones bajas e intermedias, rocas metamórficas en terrenos intermedios, rocas graníticas en terrenos altos quebrados a montañosos.

ZP-13. VALLE DE LOS COLONOS: Límites $37^{\circ} 59^{\prime} \mathrm{S} 72^{\circ} 10^{\prime} \mathrm{W}, 41^{\circ} 45^{\prime} \mathrm{S} 73^{\circ} 37^{\prime} \mathrm{W}$; depresión intermedia regiones de la Araucanía, Los Ríos y Los Lagos; altitudes entre 50 y 600 msnm; rango de precipitación media anual 1501-2000 mm; presencia de tipos forestales Roble-Raulí-Coihue, Siempreverde, Esclerófilo; descripción del suelo presente en esta zona: cenizas volcánicas sobre gravas y sobre material fluvioglaciar o aluvial.

ZP-14. VILLARRICA: Límites $37^{\circ} 59^{\prime} \mathrm{S} 72^{\circ} 08^{\prime} \mathrm{W}, 40^{\circ} 25^{\prime} \mathrm{S} 71^{\circ} 52^{\prime} \mathrm{W}$; pre cordillera y cordillera de Los Andes regiones de la Araucanía y Los Ríos; altitudes entre 600 y $1500 \mathrm{msnm}$; rango de precipitación media anual 2501-3500 mm; presencia de tipos forestales Roble-Raulí-Coihue, Coihue-Raulí-Tepa, Lenga, Araucaria y Ciprés de la Cordillera; suelos aluviales, cenizas volcánicas, textura media y fina.

ZP-15. COSTA VALDIVIA-ZARAO: Límites $39^{\circ} 18^{\prime} \mathrm{S} 72^{\circ} 51^{\prime} \mathrm{W}, 41^{\circ} 45^{\prime} \mathrm{S} 73^{\circ} 38^{\prime} \mathrm{W}$; costa regiones de Los Ríos y Los Lagos; altitudes entre 0 y $700 \mathrm{msnm}$; rango de precipitación media anual 2001-2500 mm; presencia de tipos forestales Siempreverde, Alerce, Coihue de Magallanes y Roble-Rauli-Coihue; descripción del suelo ubicado en esta zona: roca metamórfica micaesquística en terrenos de cerro y valles sedimentarios, cenizas volcánicas antiguas sobre roca metamórfica.

ZP-16. HORNOPIRÉN: Límites $40^{\circ} 21^{\prime} \mathrm{S} 72^{\circ} 27^{\prime} \mathrm{W}, 44^{\circ} 01^{\prime} \mathrm{S} 71^{\circ} 46^{\prime} \mathrm{W}$; pre cordillera y cordillera de Los Andes región de Los Lagos; altitudes entre 0 y 1200 msnm; rango de precipitación media anual $3501-4000 \mathrm{~mm}$; presencia de tipos forestales Siempreverde, Roble-Raulí-Coihue, Coihue-Raulí-Tepa, Lenga, Alerce y Ciprés de la Cordillera. Descripción de suelos: cenizas volcánicas modernas sobre arena, y escoria volcánica sobre grava pumicíticas; arena y lava andesítico-basáltica en topografía plana y ladera de cerros. ZP-17. CHILOÉ: Límites $41^{\circ} 48^{\prime} S 74^{\circ} 01^{\prime} \mathrm{W}, 43^{\circ} 25^{\prime} \mathrm{S} 73^{\circ} 48^{\prime} \mathrm{W}$; costa región de Los Lagos; altitudes entre 0 y $600 \mathrm{msnm}$; rango de precipitación media anual $2001-2500 \mathrm{~mm}$; presencia de tipos forestales Siempreverde, Alerce, Ciprés de las Guaitecas. 


\section{RESULTADOS}

La caracterización general de los ensayos Remeco y Huillilemu, en término de promedio, desviación estándar y diferencias mínimas significativas para cada variable se resume en el Cuadro $N^{\circ}$ 2. En el cuadro se destaca que el ensayo Remeco presenta mejor desempeño en supervivencia y rectitud de fuste y menor crecimiento en altura y DAP que el ensayo Huillilemu, este último es un año mayor que Remeco.

El desempeño de coigüe analizado en conjunto para Remeco y Huillilemu no evidencia diferencias estadísticamente significativas entre zonas de procedencia para supervivencia, DAP ni rectitud de fuste, solo se observan diferencias significativas para la variable altura (Figura $\mathrm{N}^{\circ} 3$ ).

Cuadro $\mathrm{N}^{\circ} 2$

CARACTERIZACIÓN GENERAL DE LOS ENSAYOS EVALUADOS

\begin{tabular}{|c|c|r|r|r|r|}
\hline Parámetro & Ensayo & $\begin{array}{c}\text { Supervivencia } \\
{[\%]}\end{array}$ & $\begin{array}{c}\text { Altura } \\
{[\mathbf{m}]}\end{array}$ & $\begin{array}{c}\text { DAP } \\
{[\mathbf{c m}]}\end{array}$ & $\begin{array}{c}\text { Rectitud } \\
{[1-4]}\end{array}$ \\
\hline \multirow{4}{*}{ Promedio } & Huillilemu & $\mathbf{7 1 , 8} \mathrm{b}$ & $\mathbf{1 5 , 4} \mathrm{a}$ & $\mathbf{1 8 , 0} \mathrm{a}$ & $\mathbf{1 , 4} \mathrm{a}$ \\
(Desviación & $\mathrm{n}=323$ & $(22,9)$ & $(2,5)$ & $(4,3)$ & $(0,5)$ \\
\cline { 2 - 6 } estándar) & Remeco & $\mathbf{8 0 , 6} \mathrm{a}$ & $\mathbf{1 4 , 9} \mathrm{b}$ & $\mathbf{1 5 , 6} \mathrm{b}$ & $\mathbf{1 , 7} \mathrm{b}$ \\
& $\mathrm{n}=290$ & $(24,3)$ & $(1,4)$ & $(3,4)$ & $(0,9)$ \\
\cline { 2 - 6 } & Conjunto & $\mathbf{7 5 , 7}$ & $\mathbf{1 5 , 2}$ & $\mathbf{1 6 , 9}$ & $\mathbf{1 , 5}$ \\
& $\mathrm{n}=613$ & $(24,2)$ & $(2,1)$ & $(4,1)$ & $(0,7)$ \\
\hline \multicolumn{2}{|l}{ DMS (Tuckey, alfa $\mathbf{0 , 0 5 )}$} & $3,48 \%$ & $0,32 \mathrm{~m}$ & $0,60 \mathrm{~cm}$ & 0,12 \\
\hline
\end{tabular}

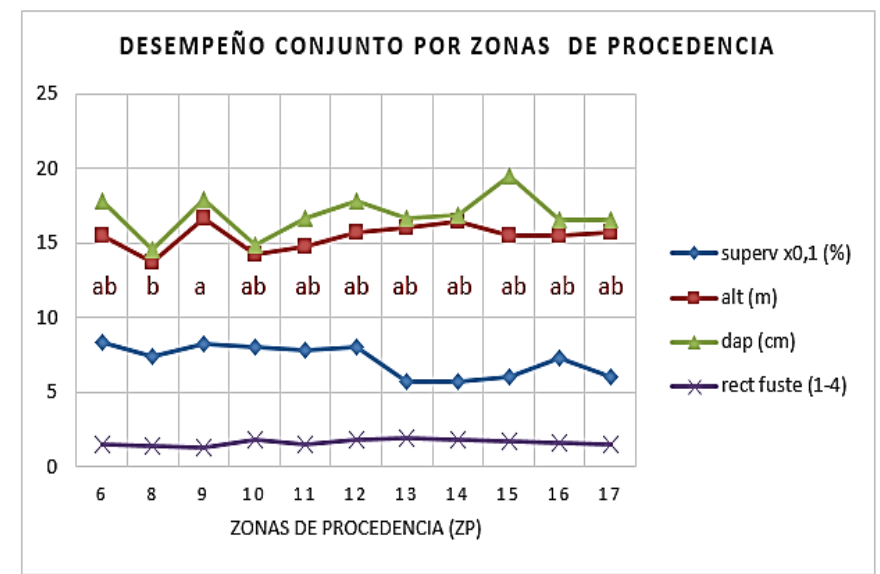

(Las letras corresponden al agrupamiento de las medias de altura para una diferencia mínima significativa de 2,692 m)

Figura $\mathrm{N}^{\circ} 3$

VARIACIÓN DE SUPERVIVENCIA, ALTURA, DAP Y RECTITUD DE FUSTE POR ZONAS DE PROCEDENCIA EN EVALUACION CONJUNTA DE DOS ENSAYOS DE PROCEDENCIAS Y PROGENIES DE COIGÜE DE 14 Y 15 AÑOS DE EDAD 
A nivel de ensayos individuales se mantiene la misma tendencia anterior, detectándose diferencias entre zonas de procedencia que solo son significativas en el caso de la variable altura del ensayo Remeco (Cuadros $N^{\circ} 3$ y $N^{\circ} 4$ y Figura $N^{\circ} 4$ ).

Cuadro $\mathrm{N}^{\circ} 3$

DESEMPEÑO DE COIGÜE POR ZONA DE PROCEDENCIA ENSAYO HUILLILEMU

\begin{tabular}{|c|r|r|r|r|r|}
\hline $\begin{array}{c}\text { Zona de } \\
\text { Procedencia }\end{array}$ & \multicolumn{1}{c|}{$\mathbf{n}$} & $\begin{array}{c}\text { Superv. } \\
\text { [\%] }\end{array}$ & $\begin{array}{c}\text { Altura } \\
\text { [m] }\end{array}$ & $\begin{array}{c}\text { DAP } \\
\text { [cm] }\end{array}$ & $\begin{array}{c}\text { Rectitud } \\
\text { Fuste } \\
{[\mathbf{1 - 4}]}\end{array}$ \\
\hline ZP 6 & 89 & 83,2 & 15,7 & 18,6 & 1,3 \\
\hline ZP 8 & 15 & 65,2 & 13,7 & 15,0 & 1,5 \\
\hline ZP 9 & 9 & 75,0 & 16,6 & 18,8 & 1,2 \\
\hline ZP10 & 4 & 80,0 & 14,2 & 14,9 & 1,7 \\
\hline ZP11 & 146 & 74,9 & 15,1 & 17,9 & 1,4 \\
\hline ZP12 & 12 & 80,0 & 15,7 & 17,9 & 1,6 \\
\hline ZP13 & 14 & 56,0 & 16,2 & 18,0 & 1,3 \\
\hline ZP14 & 12 & 42,9 & 16,8 & 17,9 & 1,5 \\
\hline ZP15 & 3 & 60,0 & 15,5 & 19,5 & 1,7 \\
\hline ZP16 & 17 & 56,7 & 16,4 & 19,2 & 1,4 \\
\hline ZP17 & 2 & 40,0 & 14,2 & 14,9 & 1,5 \\
\hline Total & $\mathbf{3 2 3}$ & $\mathbf{7 1 , 8}$ & $\mathbf{1 5 , 4}$ & $\mathbf{1 8 , 0}$ & $\mathbf{1 , 4}$ \\
\hline DMS & & $\mathbf{8 1 , 7}$ & $\mathbf{4 , 0 2}$ & $\mathbf{6 , 9 2}$ & $\mathbf{0 , 9 3}$ \\
(Tuckey, alfa $=0,05)$ & $\mathbf{0 , 0 3}$ &
\end{tabular}

Cuadro $\mathrm{N}^{\circ} 4$

DESEMPEÑO DE COIGÜE POR ZONA DE PROCEDENCIA

ENSAYO REMECO

\begin{tabular}{|c|c|c|c|c|c|}
\hline $\begin{array}{c}\text { Zona de } \\
\text { Procedencia }\end{array}$ & $\mathbf{n}$ & $\begin{array}{c}\text { Superv. } \\
{[\%]}\end{array}$ & $\begin{array}{c}\text { Altura } \\
{[\mathrm{m}]}\end{array}$ & $\begin{array}{l}\text { DAP } \\
{[\mathrm{cm}]}\end{array}$ & \begin{tabular}{|c} 
Rectitud \\
Fuste \\
{$[1-4]$}
\end{tabular} \\
\hline ZP 6 & 44 & 84,6 & $15,1 \quad a b c$ & 16,0 & 1,9 \\
\hline ZP 8 & 16 & 84,2 & $13,7 \quad c$ & 14,0 & 1,3 \\
\hline ZP 9 & 9 & 90,0 & 16,5 a & 17,0 & 1,3 \\
\hline ZP10 & -- & -- & -- & -- & -- \\
\hline ZP11 & 143 & 82,7 & 14,5 bc & 15,5 & 1,6 \\
\hline ZP12 & 4 & 80,0 & $15,7 \mathrm{ab}$ & 17,4 & 2,3 \\
\hline ZP13 & 21 & 58,3 & $15,8 \mathrm{ab}$ & 15,6 & 2,2 \\
\hline ZP14 & 17 & 73,9 & $16,1 \quad a b$ & 16,2 & 1,9 \\
\hline ZP15 & -- & -- & -- & -- & -- \\
\hline ZP16 & 32 & 86,5 & $15,1 \quad a b c$ & 15,0 & 1,7 \\
\hline ZP17 & 4 & 80,0 & 16,5 a & 17,3 & 1,5 \\
\hline Total & 290 & 80,6 & 14,9 & 15,6 & 1,7 \\
\hline $\begin{array}{c}\text { DMS } \\
\text { (Tuckey, alfa= }\end{array}$ & & 59,4 & 1,72 & 4,59 & 1,2 \\
\hline
\end{tabular}

Letras distintas en la misma columna indican diferencias estadísticamente significativas entre medias (Tuckey alfa=0,05) 


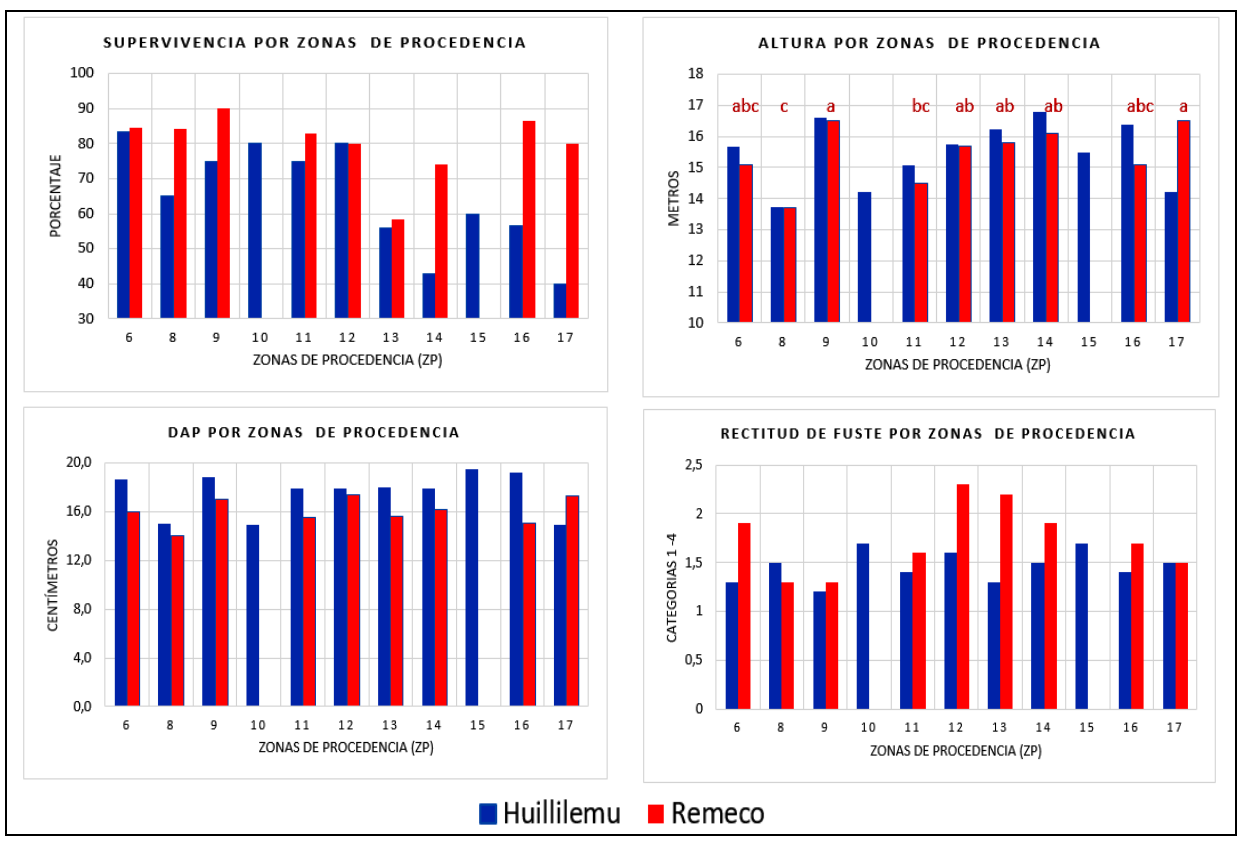

Las letras en el gráfico de altura indican grupos estadísticamente homogéneos según prueba de Tuckey $(\mathrm{Alfa}=0,05)$ para zonas de procedencia en el ensayo Remeco

Figura $N^{\circ} 4$

SUPERVIVENCIA, CRECIMIENTO Y RECTITUD DE FUSTE DE COIGÚE POR ZONA DE PROCEDENCIA EN ENSAYOS HUILLILEMU (15 AÑOS) Y REMECO (14 AÑOS)

\section{DISCUSIÓN}

\section{Desempeño General Ensayos}

Los ensayos evaluados corresponden a una edad de 14 y 15 años, coincidente con el periodo en que coigüe presentaría los mayores incrementos medios anuales. En efecto, antecedentes compilados por Donoso et al. (2007) indican que los mayores crecimientos de coigüe se producirían entre los 10 y 15 años en las plantaciones y entre los 15 y 30 años en renovales.

Los valores medios de altura $(15,4$ y $14,9 \mathrm{~m})$ y DAP $(18,0$ y $15,6 \mathrm{~cm})$ registrados en Huillilemu y Remeco, respectivamente, corresponden a incrementos medios anuales que oscilan entre 1,03 y $1,06 \mathrm{~m} / \mathrm{año}$ en altura y 1,1 a $1,2 \mathrm{~cm} / \mathrm{año}$ en diámetro. Para ambas variables los valores obtenidos se encuentran dentro de los rangos informados para otras plantaciones de coigüe, los cuales de acuerdo a antecedentes de diferentes autores compilados por Gutiérrez

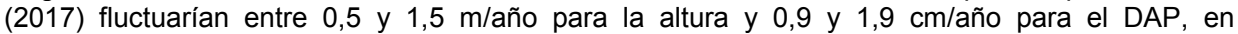
plantaciones de coigüe menores de 20 años. Así, aunque se han descrito crecimientos bastantes heterogéneos, en general se reconoce que coigüe manifiesta un crecimiento más bien rápido entre las especies arbóreas del cono sur (Cruz-Johnson, 2013). 
En cuanto a supervivencia, las plantas ensayadas en Remeco presentan mejor supervivencia $(80,6 \%)$ que las establecidas en Huillilemu $(71,8 \%)$, siendo estadísticamente significativa la diferencia entre ensayos. En ambos casos los valores son equiparables a los informados para otras plantaciones de coigüe a edades más tempranas: $75-96 \%$ después de un año de establecidas (Büchner, 2007); $90-100 \%$ en plantaciones de cuatro años (Álvarez y Lara, 2008); 69\% a los seis años (Betancurt, 2015); 86\% a los siete años (Donoso y Soto, 2010).

Respecto a rectitud de fuste los valores medios obtenidos en ambos ensayos $(1,4$ en Huillilemu y 1,7 en Remeco) son bajos y estadísticamente diferentes entre ellos. Se observa escasa presencia de árboles rectos, situación que también ha sido descrita por Betancurt (2015) en otras plantaciones de coigüe. En general la calidad de fuste no exhibe variación entre progenies y procedencias y parecería ser un atributo que junto con las bifurcaciones constituiría una característica intrínseca de la especie (Gutiérrez, 2017).

\section{Efecto de Zonas de Procedencias}

Dependiendo de si la variabilidad genética está determinada o no por fuerzas de selección, se clasifica en variabilidad genética adaptativa o neutral. Esta última, independiente de las fuerzas de selección se estima principalmente mediante marcadores moleculares, en cambio el método tradicional para estimar la variabilidad genética adaptativa es evaluando caracteres morfométricos, fenológicos o fisiológicos en ensayos de procedencias y pruebas de progenies, enfoque empleado en el presente artículo.

En los ensayos evaluados no se evidenció efecto de las zonas de procedencia sobre el desempeño del material genético en término de las variables consideradas, exceptuando el comportamiento de la variable altura en el ensayo Remeco.

Los resultados pueden parecer inesperados, especialmente si se considera que coigüe ocupa distintos sitios en 10 de los 12 tipos forestales definidos para Chile, reflejando así su adaptación a diferentes condiciones de clima y suelo (Esse et al., 2013), presenta un amplio rango de distribución desde los $34^{\circ} 37^{\prime}$ a los $47^{\circ} 30^{\prime} \mathrm{S}$, se desarrolla en una gran diversidad de ambientes, con amplitud de hábitats en sentidos latitudinal, longitudinal y altitudinal, aspectos todos que sugieren una marcada variabilidad y diferenciación entre sus poblaciones. Sin embargo, como lo indican Hasbún et al. (2014) en el caso de las extensas y relativamente continuas poblaciones de coigüe, si bien debería esperarse una elevada variación genética poblacional, existe también un elevado flujo génico en la mayor parte de su distribución, particularmente desde los $37^{\circ}$ al sur, que reduce la divergencia genética entre sus poblaciones.

Los resultados del presente estudio coinciden en identificar alta variación en el desempeño de los individuos analizados, pero esta variabilidad se presenta principalmente en las familias dentro de las zonas de procedencias y no se refleja en diferencias entre las zonas de procedencia propiamente tales. Esta misma situación, junto al efecto del flujo génico como fenómeno que reduce las diferencias genéticas entre poblaciones, ha sido demostrada en estudios de variabilidad isoenzimática de coigüe efectuados por investigadores argentinos citados en Donoso et al. (2004), donde se concluye que la mayor parte de la variación genética se encuentra dentro de las poblaciones, mientras que el porcentaje entre distintas poblaciones resulta ser muy bajo.

Estudios con herramientas moleculares confirman los resultados anteriores. Utilizando marcadores AFLP en muestras de ADN obtenidas desde diversas zonas de la distribución natural 
de coigüe se observa que la variabilidad genética de la especie en las localidades evaluadas es alta, pero no se detectan alelos únicos de localidades específicas, concluyéndose la existencia de bajos niveles de diferenciación genética poblacional o entre localidades (Hasbún et al., 2014).

En la misma línea, estudios con moderna tecnología molecular y marcadores SNP altamente polimórficos y discriminativos, utilizados para caracterizar poblaciones de coigüe, también indican una baja diferenciación genética entre poblaciones. En efecto, tras analizar muestras de ADN provenientes de más de 20 poblaciones distribuidas en los siete estratos ecológicos ambientales en que Alarcón et al. (2013) dividen la distribución de coigüe, Gonzalez (2015) concluye que los siete estratos definidos por modelamiento ecológico no constituyen grupos genéticamente diferentes, y que la estructuración genética estaría constituida solo por dos grupos a lo largo de toda la distribución geográfica de la especie. Inicialmente, Hasbun et al. (2014) también proponen a los siete estratos de Alarcón et al. (2013) como procedencias diferentes, no obstante, al integrar los resultados de sus análisis genéticos concluyen la presencia de solo 3 subpoblaciones, y que solo dos de ellas serían puras; una en el norte (estrato 1 y 2 ) y otra en el sur (estrato $3^{4}$ ), la tercera sería una mezcla de las dos anteriores (estratos 4, 5, 6 y 7). En síntesis, confirman una diferenciación genética muy inferior respecto a la esperada de la división ecológica-ambiental, equivalente a lo observado en las zonas de procedencia analizadas en este artículo.

La independencia del efecto de las zonas de procedencias observada en este estudio también ha sido descrita en otras experiencias de establecimiento de plantaciones experimentales, como en el caso de lenga (Nothofagus pumilio), donde Mondino et al. (2010) observan este mismo fenómeno entre procedencias con diferencias de altitud del orden de $400 \mathrm{msnm}$. Por otra parte, se reconoce que atributos relevantes para la caracterización forestal, entre otros la altura y el diámetro utilizados en este estudio, no suelen expresar fielmente las diferencias genéticas entre los árboles (Puntieri et al., 2006).

A partir de los resultados obtenidos y de los antecedentes bibliográficos complementarios se puede desprender que coigüe exhibe variabilidad, pero que esta se manifiesta más bien dentro de las zonas de procedencia que entre ellas; y que la variabilidad genética entre poblaciones de coigüe sería menor que la presumida en función de la amplia diversidad ambiental y ecológica que ocupa la especie.

Parte de la variación observada en las poblaciones naturales obedecería a fenómenos de plasticidad fenotípica ajenos a origen genético y por lo tanto no heredables como para observarlos en ensayos como los evaluados; este sería el caso por ejemplo de la variabilidad respecto a tamaño de semillas en un gradiente clinal de latitud, o de la variación ecotípica que separa a las poblaciones mediterráneas de la más sureñas.

Debe existir variación genética adaptativa, pero no se estaría expresando en variables como las aquí consideradas, sino que presumiblemente en otras que estén más relacionadas con la capacidad de supervivencia de los individuos, que con factores de productividad forestal. Por ejemplo, variables asociadas a la eficiencia en el transporte de agua, respecto de las cuales Carrasco-Urra et al. (2018) determinan la existencia de diferencias estadísticamente significativas entre poblaciones de coigüe ubicadas a lo largo de su distribución andina.

\footnotetext{
${ }^{4}$ En el estudio de Hasbún el estrato 3 corresponde a muestras de Coyhaique y Aysén. Estos orígenes no están representados en las procedencias y zonas de procedencia contempladas en los ensayos evaluados en este artículo. Consecuentemente, de acuerdo a la clasificación genética de Hasbún, el material evaluado en las zonas de procedencia correspondería solo a una población pura y a una mezcla, explicando la falta de diferenciación entre los grupos evaluados.
} 


\section{CONCLUSIÓN}

De acuerdo con los resultados obtenidos el origen de las semillas, en términos de las zonas de procedencia analizadas, no tendría efecto para diferenciar el desempeño en altura, diámetro, supervivencia y rectitud de fuste de coigüe. Al respecto, antecedentes complementarios citados en el texto sugieren una escasa diferenciación genética entre poblaciones, la que justificaría el resultado obtenido.

\section{REFERENCIAS}

Agúndez, D.; Martín, S.; De Miguel, J.; Galera, R.; Jiménez, M. y Díaz-Fernández, P., 1995. Las regiones de procedencia de Fagus sylvatica en España. ICONA. Madrid, España. ISBN 84-8014-139-5. 104 p.

Alarcón, D.; Fuentes, G.; Hasbún, R. y Ruíz, E., 2013. Estratificación ecológica de poblaciones de Nothofagus dombeyi en Chile para asistir el muestreo de su diversidad genética. https://www.researchgate.net/publication/304540561.

Alía, R.; Mancha, J.; Sánchez de Ron, D.; Barba, D.; Climent, J.; García del Barrio, J.; Notivol, E. e Iglesias, S., 2009a. Las regiones de procedencia de las especies forestales en Europa. En: Revista de la Asociación y Colegio Oficial de Ingenieros Técnicos Forestales, Foresta N46. Pp: 44-48.

Alía, R.; García del Barrio, J.; Iglesias, S.; Mancha, J.; de Miguel, J.; Peragón, J.; Pérez, F. y Sánchez del Ron, D., 2009b. Regiones de procedencia de especies forestales en España. CIFOR-INIA. Dpto. Sistemas y Recursos Forestales. Madrid, España. 363 p.

Álvarez, C. y Lara, A., 2008. Crecimiento de una plantación joven en fajas con especies nativas en la Cordillera de Los Andes de la provincia de Valdivia. Bosque Nativo 29 (3): 181-191.

Azpilicueta, M.; Marchelli, P.; Umaña, F.; Thomas, E.; Van Zonneveld, M. y Gallo, L., 2016. Definición de zonas genéticas como fuente de semilla en raulí y roble pellín en Argentina. En: Azpilicueta, M. y Marchelli, P. (Eds). Zonas genéticas de raulí y roble pellín en Argentina: Herramienta para la conservación y el manejo de la diversidad genética. INTA Ediciones. Bariloche, Rio Negro. Pp: 21-28

Betancurt, G., 2015. Evaluación del desempeño de una plantación joven de coihue establecida a campo abierto en el predio Llancahue, comuna de Valdivia. Trabajo de Titulación presentado como parte de los requisitos para optar al Título de Ingeniero Forestal. Facultad de Ciencias Forestales y recursos naturales. Universidad Austral de Chile. Valdivia. $61 \mathrm{p}$.

Büchner, C., 2007. Respuesta inicial de una plantación de Nothofagus dombeyi (Mirb) Oerst a distintas dosis de fertilizante, en la precordillera de la Costa de Valdivia. Tesis Escuela de Ingeniería Forestal, Facultad de Ciencias Forestales, Universidad Austral de Chile. Valdivia, Chile. http://cybertesis.uach.cl/tesis/uach/2007/fifb919r/doc/fifb919r.pdf.

Carrasco-Urra, F.; Quezada, I. y Saldaña, A., 2018. Variation in traits related to wáter transport in Nothofagus dombeyi helps to explain its latitudinal distribution limit in the Chilean Andes. Plant Ecology \& Diversity, DOI: 10.1080/17550874.2018.1507056.

Cruz-Johnson, P., 2013. Aplicación de relaciones de densidad-diámetro y de área potencial aprovechable en bosques de Nothofagus dombeyi (Mirb.) Oerst, como herramienta de planificación silvícola. Tesis doctoral Departamento de Ciencia y Tecnología Agroforestal y Genética. Universidad de Castilla La Mancha. Albacete, España. 120 p.

Donoso, P. y Soto, D., 2010. Plantaciones con especies nativas en el centro-sur de Chile: experiencias, desafíos y oportunidades. Bosque Nativo $\mathrm{N}^{\circ} 47$, julio-octubre 2010, pp: 10-17. 
Donoso, P.; Soto, D.; Donoso, C. y Rivera, H., 2007. Crecimiento inicial en altura de plantaciones de coihue, roble y raulí en altitudes medias en la provincia de Valdivia, Chile. Bosque Nativo N 40. Dic 2006 - Abr 2007 . Pp: 8-12.

Donoso, C.; Premoli, A. y Donoso, P., 2004. Variación en Nothofagus siempreverdes sudamericanos. En: Donoso, C., Premoli, A.; Gallo, L. e Ipinza, R. (editores) Variación Intraespecífica en las especies arbóreas de los bosques templados de Chile y Argentina. Editorial Universitaria. Santiago, Chile. Capítulo 8, pp: 189-214.

Esse, C.; Donoso, P.; Gerding, V. y Encina-Montoya, F., 2013. Determination of homogeneous edaphoclimatic zones for the secondary forest of Nothofagus dombeyi in central southern Chile. Cien. Inv. Agrar. 40(2):351-360.

Esse, C.; Donoso, P.; Gerding, V.; Navarro, C. y Encina-Montoya, F., 2014. Modelling dominant height and site index in different edaphoclimatic zones of Nothofagus dombeyi secondary forest in the Andes of south-central Chile. Southern Forest 2014:1-8

García, L.; Droppelmann, F. y Rivero, M., 2013. Morfología y fenología floral de Nothofagus alpina (Nothofagaceae) en un huerto semillero clonal en la región de Los Ríos, Chile. En: Bosque 34(2):221-231.

García del Barrio, J.; De Miguel, J.; Alía, R. e Iglesias, S., 2001. Regiones de identificación y utilización de material forestal de reproducción. Serie cartográfica. Secretaría general del medio ambiente. Dirección General de Conservación de la Naturaleza. España. 293 p.

González, J., 2015. Estructura genética y detección de loci bajo selección en Nothofagus dombeyi (Mirb) Oerst. mediante datos moleculares obtenidos por genotyping by sequencing (GBS). Tesis para optar al título de Ingeniero en Biotecnología Vegetal. Facultad de Cs Forestales. Universidad de Concepción. Chile. 55p.

Gutiérrez, B., 2019. Regiones de procedencias, un ordenamiento de fuentes semilleras. Ciencia e Investigación Forestal 25(2):57-73.

Gutiérrez, B., 2017. Evaluación de crecimiento y forma de fuste de un ensayo de procedencias y progenies de coihue (Nothofagus dombeyi (Mirb.) Oerst.) de 15 años de edad. Ciencia e Investigación Forestal 23(3): 31-42.

Hasbún, R.; Ruiz, E.; Ríos, D.; Fuente, G y Alarcón, D., 2014. Certificación genética del origen de materiales reproductivos de coigüe mediante herramientas moleculares y nichos ecológicos. Informe Final Proyecto FIBNCONAF 068/2012. Universidad de Concepción, Concepción. Octubre 2014. 59 p.

Marchelli, P.; Azpilicueta, M.; Thomas, E.; Van Zonneveld, M. y Gallo, L., 2016. Distribución futura: Impacto del cambio climático global. En: Azpilicueta, M. y Marchelli, P. (Eds). Zonas genéticas de raulí y roble pellín en Argentina: Herramienta para la conservación y el manejo de la diversidad genética. INTA Ediciones. Bariloche, Rio Negro. Pp: 35-38.

Mondino, V.; Tejera, L.; Pastorino, M. y Gallo, L., 2010. Establecimiento en Nothofagus pumilio: efecto de plantas nodrizas y procedencias. Poster. Estación Experimental Agroforestal Esquel, Centro Regional Patagonia Sur, INTA Argentina.

Pastorino, M. y Mondino, V., 2016. Restauración de bosques nativos: La importancia del origen de las semillas. Actualidad El Cordillerano. 5 septiembre, 2016.

Puntieri, J.; Grosfeld, J.; Stecconi, M.; Brion, C.; Azpilicueta, M. y Gallo, L., 2006. Early development of roble (Nothofagus obliqua), an architectural analysis of provenances from Argentina. Bosque 27(1):44-51.

Quiroz, I. y Gutiérrez, B., 2014. Definición de zonas de procedencia para especies el bosque nativo. En: Propuesta de reglamento para semillas y plantas forestales. INFOR-SAG. Concepción, Chile. 74 p.

Teillier, S.; Macaya-Berti, J.; Bonnemaison, C.; Delaunoy, J. y Marticorena, A., 2013. Contribución al conocimiento de la flora vascular de la Reserva Biológica Huilohuilo, Región de Los Ríos, Chile. Gayana Botanica 70(2): 194-234. 
